\title{
L-Arginine Pathway Metabolites Predict 6 Months Outcome after Acute
} Ischemic Stroke

\section{Peter Csecsei ${ }^{1 *}$, Lajos Nagy², Sandor Keki², Laszlo Szapary ${ }^{1}$, Zsolt Illes ${ }^{1,3,4}$, Nelli Farkas ${ }^{5}$ and Tihamer Molnar}

${ }^{1}$ Department of Neurology, Medical School, University of Pecs, Pecs, Hungary

${ }^{2}$ Department of Applied Chemistry, University of Debrecen, Debrecen, Hungary

${ }^{3}$ Department of Neurology, Odense University Hospital, Odense, Denmark

${ }^{4}$ Institute of Clinical Research, University of Southern Denmark, Odense, Denmark

5 Institute of Bioanalysis, Medical School, University of Pecs, Pecs, Hungary

${ }^{6}$ Department of Anaesthesiology and Intensive Care, Medical School, University of Pecs, Pecs, Hungary

\begin{abstract}
Background: Increased levels of asymmetric dimethylarginine (ADMA) and symmetric dimethylarginine (SDMA) are associated with endothelial dysfunction. Here, we analyzed the relationship between L-arginine pathway metabolites and functional outcome of ischemic stroke at discharge and 6 months follow-up.
\end{abstract}

Methods: Plasma concentration of L-arginine, ADMA, SDMA were investigated in 46 patients at post-stroke 24 h. Outcome measures were assessed by modified Rankin scale (mRS) at hospital discharge and 6 months follow-up. According to change in mRS score during the 6 months period, patients were divided into subgroups of improved, unchanged and worsened function. Predictive role of L-arginine pathway metabolites were explored in these outcome groups.

Results: Significant inverse correlations were found between initial NIHSS and the L-arginine/ADMA, the $\mathrm{L}$-arginine/SDMA ratios and L-arginine plasma concentration (all $p<0.05$, respectively). Patients with worsened $m R S$ by 6 months had significantly higher L-arginine plasma concentrations at 24 post-stroke hours compared to patients with improved mRS $(p<0.001)$ and unchanged mRS $(p<0.005)$. The L-arginine/ADMA $(p<0.004)$ and the L-arginin/ SDMA $(p<0.002)$ ratios at $24 \mathrm{~h}$ were significantly higher among patients with worsened compared to improved $\mathrm{mRS}$. Besides, clinical factors, such as BMI showed negative correlation with L-arginine/ADMA; creatinine showed positive correlation with L-arginine and L-arginine/ADMA; LDL showed positive correlation with L-arginine/SDMA ratio. Plasma concentration of ADMA was significantly higher among smokers compared to non-smokers. The L-arginine/ADMA, the L-arginine/SDMA ratios and L-arginine plasma concentration negatively correlated with change of mRS between hospital discharge and at 6 months.

Conclusion: Our data indicate that lower L-arginine/SDMA ratio is associated with worse outcome on the shortterm, but these patients improve on the long-term resulting in association of lower ratios with improving mRS. In contrast, patients with high L-arginine and low mRS improve fast, but will not improve or may even worsen by 6 months indicating a time-dependent biological effect.

Keywords: L-arginine pathway; Acute ischemic stroke; ADMA; SDMA

\section{Introduction}

Stroke is the third most common cause of disability worldwide [1]. Costs to treat stroke are projected to more than double and the number of people having strokes may increase $20 \%$ by 2030 , according to the American Heart Association/American Stroke Association. These cost projections for stroke are comparable to those for other high-impact chronic diseases, such as cancer and cardiovascular disease [2].

Prognostic tools that monitor the neurological condition and predict the final outcome in clinical practice are scarce. Although several blood biomarkers have been proposed as promising biomarkers, e.g. C-reactive protein (CRP), tumor necrosis factor (TNF), interleukin-6 (IL-6) and CD40L, no biomarker is used in clinical practice [3].

Nitric oxide (NO) plays a role in maintaining vascular integrity [4] NO is synthetized by the oxidation of L-arginine, which can be inhibited by asymmetric dimethylarginine (ADMA). Symmetric dimethylarginine (SDMA) competes with arginine uptake and antagonizes the effects of L-arginine [5]. The plasma concentration of L-arginine and its dimethylated derivatives ADMA and SDMA were associated with longterm mortality in patients followed up to 7 years after an acute stroke [6]. SDMA level in the plasma was strongly associated with adverse clinical outcome during the first 30 days after ischemic stroke [7]. Increased plasma levels of both ADMA and SDMA within the first $72 \mathrm{~h}$ after onset of acute ischemic stroke predict poor functional outcome 90 days after stroke onset [8].

The aim of the present study was to investigate the relationship between early L-arginine and dimethylarginine plasma levels and outcome of ischemic stroke at discharge and 6 months later in a prospective study. Moreover, we explored whether L-arginine pathway metabolites predict worsening of 6 months clinical outcome compared to baseline.

\section{Materials and Methods}

\section{Study population}

The study was approved by regional ethics committee and patients or their legal representatives gave informed consent.

*Corresponding author: Peter Csecsei, Department of Neurology, University of Pecs, Pecs, Hungary, Tel: 3672535900; Fax: 3672535911; E-mail: csecseipeti@yahoo.com

Received April 24, 2018; Accepted April 28, 2018; Published April 30, 2018

Citation: Csecsei P, Nagy L, Keki S, Szapary L, Illes Z, et al. (2018) L-Arginine Pathway Metabolites Predict 6 Months Outcome after Acute Ischemic Stroke. Int J Neurorehabilitation 5: 315. doi: 10.4172/2376-0281.1000315

Copyright: $\odot 2018$ Csecsei $\mathrm{P}$, et al. This is an open-access article distributed unde the terms of the Creative Commons Attribution License, which permits unrestricted use, distribution, and reproduction in any medium, provided the original author and source are credited. 
Between January 2015 and April 2016, 46 patients with acute ischemic stroke were prospectively enrolled. Patients underwent systemic thrombolysis according to international guideline [9] or received conservative treatment according to recent ESC Guideline of acute ischemic stroke [10].

The etiology of stroke was classified according to Trial of ORG 10172 in acute stroke treatment (TOAST) criteria [11]. Neuroimaging was performed by Siemens SOMATOM Definition 64 Slice CT Scanner at admission.

\section{Comorbidities}

A history of hypertension was defined as the use of antihypertensive drugs or blood pressure more than $140 / 90 \mathrm{~mm} \mathrm{Hg}$ on at least two separate occasions. Diabetes mellitus was defined as the use of antidiabetic drugs or a fasting plasma glucose value $\geq 7.0 \mathrm{mmol} / \mathrm{L}$. An evidence-based guideline was followed to detect infectious complications: in short, physical and laboratory measures including white blood count (WBC), high sensitivity C-reactive protein (hsCRP), procalcitonin, fever, abnormal urine, chest $\mathrm{X}$-ray or positive cultures [12]. Body mass index (BMI) equal to or below and above $26.3 \mathrm{~kg} / \mathrm{m}^{2}$ were classified as "low BMI" and "high BMI" based on a large cohort of a similar high risk population [13].

\section{Outcome measures}

To define the size of infarct, we dichotomized Alberta stroke program early CT scores (ASPECTS) [14]: larger area involved: 0-7 or smaller area involved: 8-10 scores.

The severity of stroke was measured by the National Institute of Health Stroke Scale (NIHSS) on admission. NIHSS 0-1 was considered as mild stroke, 2-8 as moderate stroke and $\geq 9$ as a severe stroke [15].

We also recorded the modified Rankin scale (mRS) at discharge and 6 month after the onset of stroke: score $0-1$ was considered as reflecting good recovery, 2-4 as moderate recovery and 5-6 as poor recovery. Patients were sorted into 3 distinct groups based on the difference between the mRS at discharge and 6 month after stroke: (i) improved, if mRS after 6 months was lower than mRS at discharge; (ii) unchanged, if mRS after 6 months persisted; (iii) and worsened, if mRS after 6 months was higher than at discharge. To assess the mRS after 6 months, we either requested hospital visit or conducted a phone interview with the patient, the spouse, or the general practitioner.

Exclusion criteria were historical mRS higher than 0 on admission, (functional deficits prior to index event), history of malignant tumor, chronic renal failure (estimated glomerular filtration rate, eGFR $<50$ and/or creatinine $>120 \mu \mathrm{mol} / \mathrm{l}$ at two distinct measurement), any known immunological disturbances, severe hypoxia, systemic infection or sepsis.

\section{Blood collection}

Plasma samples were collected from venous blood $24 \mathrm{~h}$ after admission. The samples were immediately centrifuged (Clinspin Horizon 853VES Centrifuge) at $3000 \mathrm{rpm} / \mathrm{min}$ for $15 \mathrm{~min}$. The supernatant was stored at $-80^{\circ} \mathrm{C}$ until analysis. L-arginine, ADMA and SDMA were measured in the plasma by high-performance liquid chromatography as described previously [16].

\section{Statistical analysis}

Statistical analyses were performed using SPSS Statistics version 20.0 (IBM, 35 Armonk, NY, USA). Categorical data were summarized by means of absolute and relative frequencies (counts and percentages). Quantitative data were presented as mean and 95\% confidence interval, as well as mean \pm SD. The distribution of values was assessed by using the Shapiro-Wilk test. Non-normally distributed data are presented as median and interquartile range. Due to normally distributed data, parametric methods (chi-square test for categorical data, Student- $t$ test for continuous data) were used for demographic and clinical data. Comparison of the variables between the subgroups was performed by using the Kruskal-Wallis test, because these parameters do not follow the normal distribution. Correlation analysis was performed calculating Spearman's correlation coefficient (r). A $p$-value $<0.05$ was considered statistically significant.

\section{Results}

\section{Demographics}

Based on the change of mRS between hospital discharge and 6 months after stroke onset, patients were categorized as improved $(33 \%$, $\mathrm{n}=15)$, unchanged $(41 \%, \mathrm{n}=19)$ or worsened $(26 \%, \mathrm{n}=12)$. There were no significant differences in gender and age among these three outcome groups. Demographic and clinical characteristics of total study population and the three outcome groups based on mRS are shown in Table 1.

The median NIHSS score on admission was 6 (mean: $6.3 \pm 4.8$ ). According to NIHSS classification, $15 \%$ of the ischemic stroke was mild (NIHSS 0-1, $\mathrm{n}=6$ ), 61\% moderate (NIHSS 2-8, $\mathrm{n}=29$ ) and $24 \%$ severe (NIHSS $\geq 9, n=11)$. Two-third of the patients $(n=28,61 \%)$ had atrial fibrillation prior to stroke, 14 (30\%) had a previous ischemic heart disease or myocardial infarction and $89 \%$ of the patients had

\begin{tabular}{|c|c|c|c|c|c|}
\hline & $\begin{array}{c}\text { Patients } \\
n=46\end{array}$ & $\begin{array}{c}\text { Improved } \\
n=15\end{array}$ & $\begin{array}{c}\text { Unchanged } \\
n=19\end{array}$ & $\begin{array}{c}\text { Worsened } \\
n=12\end{array}$ & $p$ value \\
\hline Age, years & $66.8 \pm 8.4$ & $65.5 \pm 8.7$ & $67.6 \pm 8.5$ & $66.8 \pm 8.5$ & 0.470 \\
\hline Female, n (\%) & $20(43)$ & $8(53)$ & $8(42)$ & $5(42)$ & 0.506 \\
\hline Hypertension, n (\%) & $41(89)$ & $13(87)$ & $17(89)$ & $11(92)$ & 0.868 \\
\hline Diabetes, n (\%) & $10(21.7)$ & $4(27)$ & $3(16)$ & $3(25)$ & 0.856 \\
\hline Smoking, n (\%) & $21(45.6)$ & $5(33)$ & $12(63)$ & $4(33)$ & 0.844 \\
\hline BMI & $28.3 \pm 3.9$ & $27.3 \pm 4.9$ & $28.8 \pm 3.6$ & $28.5 \pm 3.6$ & 0.726 \\
\hline Creatinine, $\mu \mathrm{mol} / \mathrm{L}$ & $78 \pm 22$ & $87 \pm 35$ & $78 \pm 17$ & $70 \pm 17$ & 0.082 \\
\hline WBC, G/L & $9.1 \pm 3$ & $9.2 \pm 3$ & $9.7 \pm 4$ & $8.1 \pm 2$ & 0.371 \\
\hline CRP, mg/l & $9.1 \pm 13$ & $5.1 \pm 5$ & $11.8 \pm 18$ & $8.8 \pm 8$ & 0.338 \\
\hline Stroke severity, n (\%) & & & & & 0.269 \\
\hline Mild & $15(6)$ & $7(1)$ & $16(3)$ & $16(2)$ & \\
\hline Moderate & $61(29)$ & $67(10)$ & $58(11)$ & $67(8)$ & \\
\hline Severe & $24(11)$ & $26(4)$ & $26(5)$ & $17(2)$ & \\
\hline Discharge $\mathrm{mRS}$ score & $2.11 \pm 1.73$ & $2.73 \pm 1.4$ & $1.84 \pm 1.8$ & $1.75 \pm 1.9$ & 0.078 \\
\hline 6-month mRS score & $2.11 \pm 2$ & $1.2 \pm 1.2$ & $1.84 \pm 1.8$ & $3.67 \pm 2.3$ & 0.004 \\
\hline ASPECT score & $8.7 \pm 1.6$ & $8.8 \pm 1.4$ & $8.7 \pm 1.7$ & $8.4 \pm 1.8$ & 0.640 \\
\hline Stroke etiology, n (\%) & & & & & 0.990 \\
\hline Cardioembolic & $25(54)$ & $8(53)$ & $10(53)$ & 7 (58) & \\
\hline Atherothrombotic & $8(17)$ & $4(27)$ & $3(16)$ & 1 (9) & \\
\hline Lacunar & $9(20)$ & $1(7)$ & $5(26)$ & $3(25)$ & \\
\hline Undetermined & $4(9)$ & $2(13)$ & 1 (5) & $1(8)$ & \\
\hline Pulmonary, n (\%) & $9(19)$ & $4(27)$ & $2(11)$ & $4(33)$ & 0.608 \\
\hline Thrombolysis, n (\%) & $16(35)$ & $8(53)$ & $3(16)$ & $4(33)$ & 0.402 \\
\hline
\end{tabular}

BMI: Body Mass Index; WBC: White Blood Cells; CRP: C-Reactive Protein; ASPECT: Alberta Stroke Program Early CT Score

Data are mean $\pm S D$ and number of cases (percentage). Outcome was measured by change of modified Rankin score (mRS) between hospital discharge and 6 months after the stroke. Pulmonary include history of COPD or severe asthma

Table 1: Demographic and clinical characteristics of stroke patients on admission according to outcome after 6 months. 
hypertonia. Twenty-one subjects (46\%) were active smoker. According to TOAST criteria, $54 \%$ of the stroke was of cardioembolic origin, $17 \%$ was caused by large artery atherosclerosis, $20 \%$ was lacunar and $9 \%$ was undetermined. Sixteen patients (35\%) underwent thrombolysis and 30 (65\%) had conservative treatment.

\section{L-arginine metabolite levels at 24 post-stroke hours}

The median plasma concentration of L-arginine was $56.1 \mu \mathrm{mol} / 1$ (IQR: 41.7-71.7), the median ADMA level 0.74 $\mu \mathrm{mol} / \mathrm{l}$ (0.56-0.99), the median SDMA level $0.58 \mu \mathrm{mol} / \mathrm{l}(0.5-0.75)$, the median L-arginine/ ADMA ratio 74.1 (53.5-90.0) and the median L-arginine/SDMA ratio $88.1(57.6-130.9)$.

\section{L-arginine metabolite levels at 24 post-stroke in different severity and outcome groups}

In the three stroke severity groups based on NIHSS at admission, the median L-arginine/ADMA ratio was significantly different (mild group: median: 88.7 IQR: 78-152; moderate group: 67.8, 48-87; and severe group: 60.1, 41-80, $\mathrm{p}<0.05)$. L-arginine, ADMA, SDMA and L-arginine/SDMA showed no significant difference between the groups.

None of the examined L-arginine metabolites or ratios showed any significant correlation with either short-term outcomes (discharge $\mathrm{mRS}$ ) or long-term outcomes (6 months mRS).

Patients with worsened mRS by 6 months had significantly higher L-arginine plasma concentrations at 24 post-stroke hours compared to patients with improved mRS $(\mathrm{p}<0.000)$ and unchanged $\mathrm{mRS}$ $(\mathrm{p}<0.005)$. The L-arginine/ADMA and the L-arginine/SDMA ratios at $24 \mathrm{~h}$ were significantly higher among patients with worsened compared to improved mRS $(\mathrm{p}<0.004$ and $\mathrm{p}<0.002$, respectively). The plasma concentration of L-arginine and L-arginine/SDMA ratio was also significantly higher in the unchanged compared to the improved mRS group ( $\mathrm{p}<0.005$ and $\mathrm{p}<0.006$, respectively) (Table 2 ).

\begin{tabular}{|c|c|c|c|}
\hline & $\begin{array}{c}\text { Improved } \\
(n=15)\end{array}$ & $\begin{array}{l}\text { Worsened } \\
(n=12)\end{array}$ & $p$-value \\
\hline ADMA $(\mu \mathrm{mol} / \mathrm{l})$ & $0.58(0.46-0.8)$ & $0.7(0.59-1.04)$ & NS \\
\hline SDMA $(\mu \mathrm{mol} / \mathrm{l})$ & $0.56(0.49-0.73)$ & $0.61(0.51-0.83)$ & NS \\
\hline $\mathrm{L}$-arginine $(\mu \mathrm{mol} / \mathrm{l})$ & $38.9(25.2-47.9)$ & 79 (64.6-93.5) & $<0.000$ \\
\hline L-arginine/ADMA & $55.3(40.9-76.4)$ & $102.8(68.8-151.9)$ & $<0.004$ \\
\hline \multirow[t]{2}{*}{ L-arginine/SDMA } & $53.2(39.8-77.3)$ & $131.4(89.5-166.9)$ & $<0.002$ \\
\hline & $\begin{array}{c}\text { Improved } \\
(n=15)\end{array}$ & $\begin{array}{l}\text { Unchanged } \\
(n=19)\end{array}$ & \\
\hline ADMA $(\mu \mathrm{mol} / \mathrm{l})$ & $0.58(0.46-0.8)$ & $0.87(0.72-1.02)$ & NS \\
\hline SDMA $(\mu \mathrm{mol} / \mathrm{l})$ & $0.56(0.49-0.73)$ & $0.59(0.51-0.77)$ & NS \\
\hline L-arginine $(\mu \mathrm{mol} / \mathrm{l})$ & $38.9(25.2-47.9)$ & 57.7 (50.6-76) & $<0.005$ \\
\hline L-arginine/ADMA & $55.3(40.9-76.4)$ & 74.6 (59.6-84.9) & NS \\
\hline \multirow[t]{2}{*}{ L-arginine/SDMA } & $53.2(39.8-77.3)$ & $95.2(77.6-132.1)$ & $<0.006$ \\
\hline & $\begin{array}{c}\text { Worsened } \\
(n=12)\end{array}$ & $\begin{array}{l}\text { Unchanged } \\
(n=19)\end{array}$ & \\
\hline $\begin{array}{l}\text { ADMA }(\mu \mathrm{mol} / \mathrm{l}) \\
\text { SDMA }(\mu \mathrm{mol} / \mathrm{l}) \\
\text { L-arginine }(\mu \mathrm{mol} / \mathrm{l}) \\
\text { L-arginine/ADMA } \\
\text { L-arginine/SDMA }\end{array}$ & $\begin{array}{c}0.7(0.59-1.04) \\
0.61(0.51-0.83) \\
79(64.6-39.5) \\
102.8(68.8-151.9) \\
131.4(89.5-166.9)\end{array}$ & $\begin{array}{c}0.87(0.72-1.02) \\
0.59(0.51-0.77) \\
57.7(50.6-76) \\
74.6(59.6-84.9) \\
95.2(77.6-132.1)\end{array}$ & $\begin{array}{l}\text { NS } \\
\text { NS } \\
\text { NS } \\
\text { NS } \\
\text { NS }\end{array}$ \\
\hline
\end{tabular}

Data are shown as median $\left(25^{\text {th }}-75^{\text {th }}\right.$ percentiles). Outcome was measured by change of $\mathrm{mRS}$ between hospital discharge and 6 months after the stroke

Table 2: Plasma concentration of L-arginine pathway metabolites and ratios in different outcome groups 6 months after stroke.

\begin{tabular}{|l|c|c|c|c|c|}
\hline Variable & ADMA & SDMA & L-arginine & $\begin{array}{c}\text { L-arginine/ } \\
\text { ADMA }\end{array}$ & $\begin{array}{c}\text { L-arginine/ } \\
\text { SDMA }\end{array}$ \\
\hline Age & -0.202 & 0.006 & -0.198 & -0.168 & -0.242 \\
\hline BMI & 0.226 & 0.187 & -0.134 & $-0.295^{\star}$ & -0.135 \\
\hline Creatinine & -0.029 & 0.169 & $0.303^{\star}$ & $0.299^{*}$ & 0.149 \\
\hline Smoking & $0.419^{\star *}$ & 0.284 & 0.104 & -0.192 & -0.071 \\
\hline NIHSS & 0.130 & 0.077 & -0.266 & $-0.321^{*}$ & $-0.320^{*}$ \\
\hline ASPECT score & -0.095 & -0.082 & 0.058 & 0.055 & 0.130 \\
\hline Glucose & -0.009 & 0.141 & -0.094 & -0.073 & -0.199 \\
\hline WBC & 0.125 & 0.073 & 0.215 & 0.199 & 0.221 \\
\hline hsCRP & -0.034 & -0.094 & -0.136 & -0.101 & -0.080 \\
\hline NLR & -0.206 & 0.029 & -0.155 & 0.015 & -0.185 \\
\hline MPV & -0.046 & -0.115 & -0.047 & -0.024 & -0.059 \\
\hline Cholesterine & -0.248 & -0.169 & 0.021 & 0.189 & 0.116 \\
\hline LDL & -0.102 & -0.165 & 0.252 & 0.278 & $0.340^{*}$ \\
\hline HDL & -0.086 & -0.156 & -0.223 & -0.187 & -0.121 \\
\hline mRS discharge & 0.052 & 0.144 & -0.211 & -0.216 & $-0.326^{*}$ \\
\hline mRS 6 month & 0.246 & 0.254 & $0.312^{*}$ & 0.080 & 0.070 \\
\hline MmRS & -0.205 & -0.071 & $-0.672^{* *}$ & $-0.447^{* *}$ & $-0.517^{* *}$ \\
\hline
\end{tabular}

BMI: Body Mass Index; NIHSS: National Institute of Health Stroke Scale; ASPECT: Alberta Stroke Program Early CT Score; TOAST: Trial of Org 10172 in Acute Stroke Treatment Criteria; WBC: White Blood Cell; hsCRP: High Sensitivity C-Reactive Protein; NLR: Neutrophil-Lymphocyte Ratio; MPV: Mean Platelet Volume; LDL: Light Density Lipoprotein; HDL: High-Density Lipoprotein; mRS: Modified Rankin Score; $\triangle \mathrm{mRS}$ : mRS Discharge-Mrs

6 months all markers were measured at $24 \mathrm{~h}$ after stroke. Values are Spearman correlation coefficients

${ }^{*} \mathrm{p}<0.05,{ }^{* *} \mathrm{p}<0.01$

Table 3: Correlation among clinical data, biomarker variables and L-arginin pathway metabolites in cross-sectional analysis.

\section{Correlation of L-arginine pathway metabolites with clinical and risk factors}

ADMA, L-arginine and L-arginine/ADMA and L-arginine/SDMA ratios in the acute phase correlated with various factors (clinical and plasma molecular markers) in cross-sectional analysis (Table 3). Plasma concentration of ADMA was significantly higher among smokers compared to non-smokers (median: 0.91, IQR: 0.7-1.1 vs. 0.64, 0.5-0.82, $\mathrm{p}<0.004$ ). Higher L-arginine concentration was associated with higher creatinine concentration. Higher L-arginine/ADMA and L-arginine/ SDMA ratios were associated with lower NIHSS at admission $(-0.321$, $\mathrm{p}=0.029,-0.320, \mathrm{p}=0.03$, respectively). Higher L-arginine/ADMA ratio was also associated with lower BMI and higher creatinine concentration. Higher L-arginine/SDMA ratio was significantly associated with higher LDL levels on admission and lower discharge mRS. No correlation was found between $\mathrm{L}$-arginine metabolites and the size of infarct defined by the ASPECT scores, hsCRP, WBC, neutrophil/lymphocyte ratio, mean platelet volume, glucose, HDL and cholesterol.

We also correlated L-arginine concentration, L-arginine/SDMA and L-arginine/ADMA ratio measured $24 \mathrm{~h}$ after the onset of stroke with changes in mRS after 6 months. All of these values negatively correlated with change of mRS between hospital discharge and at 6 months ( $\triangle \mathrm{mRS}$ ) (Figure 1).

\section{L-arginine pathway metabolites measured at $24 \mathrm{~h}$ and treatment}

L-arginine/ADMA (median: 77, IQR: 61-100 vs. 52, 40-78, p=0.022) as well as L-arginine/SDMA ratio (median: 98 , IQR: 71-133 vs. 64 , 36$94, \mathrm{p}=0.019$ ) was significantly higher in the conservative treatment vs. thrombolysis group. Although, patients treated with thrombolysis had a higher baseline NIHSS than those without thrombolysis (median: 


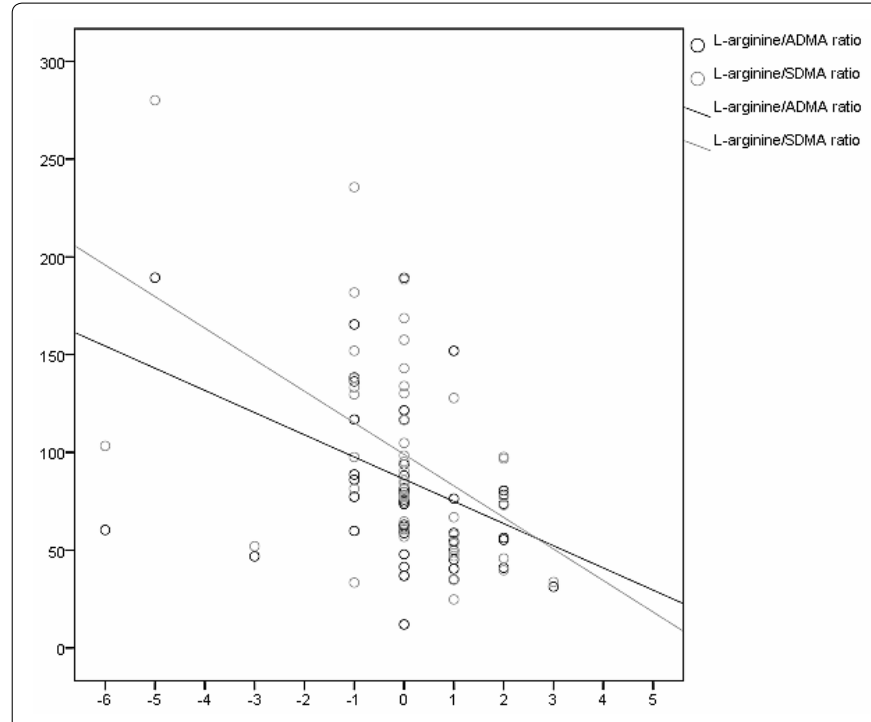

Figure 1: Correlation of dimethylarginine ratios and $\mathrm{mRS}$ change. $\triangle \mathrm{mRS}$ : mRS discharge-mRS 6 months. All markers were measured at $24 \mathrm{~h}$ after stroke.

ADMA: Asymmetric Dimethylarginine; SDMA: Symmetric Dimethylarginine Values are Spearman correlation coefficients

7.7, IQR: 5.5 - 10 vs. $4,3.7-7.5, \mathrm{p}=0.103$ ), the 6-month follow-up mRS score of patients, either receiving thrombolysis or not, was very similar (median: 2, IQR: $1.1-3.4$ vs. $1,1.3-2.7, \mathrm{p}=0.737$ ). No patients had undergone mechanical thrombectomy.

\section{Discussion}

In this study, first we explored association of the L-arginine pathway metabolites with clinical and risk factors, as well as with stroke severity, short-term outcome (mRS at discharge) and molecular markers in the acute phase of ischemic stroke. In a prospective part of the study, we investigated the plasma concentration of L-arginine pathway metabolites in 46 patients with improved, unchanged or worsened mRS by 6 months after acute ischemic stroke.

High BMI showed significant negative association with L-arginine/ ADMA ratio measured after $24 \mathrm{~h}$. Obesity is associated with elevated level of NO and markers of nitrosative and oxidative stress [13]. This may be reflected by the lower L-arginine/ADMA ratio. The higher hsCRP in patients with high BMI in our cohort presumably reflects the chronic low grade inflammation. L-arginine/SDMA ratio and SDMA at $72 \mathrm{~h}$ were independently associated with post-stroke infections [17]. Both L-arginine/ADMA and L-arginine/SDMA ratios were significantly higher in patients with conservative treatment compared to those underwent thrombolysis. Importantly, patients with higher baseline NIHSS in the thrombolysis group reached the same mRS score at 6 months follow-up compared to the conservative treatment group indicating the efficiency of recanalization therapy. Significant increase of L-arginine serum level was noticed at immediately after rtPA administration in comparison to patients treated with conservative therapy [18]. Stroke patients who were treated with rtPA showed significantly lower ADMA levels than patients who received placebo [19]. These findings are in line with our results.

Interestingly, ADMA per se was unrelated to the mRS endpoints at discharge, after 6 months and change of mRS by 6 months. It is in accordance with others findings. Schulze et al. suggested that ADMA has a less important role in stroke outcome than in primary cardiovascular disease [6]. Accordingly, ADMA was poorly associated with laboratory parameters and clinical factors in this cohort. In contrast, SDMA and L-arginine/SDMA ratio showed strong association with atrial fibrillation in our study. Similarly, SDMA, but not ADMA was closely associated with the presence of cardio-embolic stroke [20]. Higher concentration of SDMA was observed in participants with atrial fibrillation compared to patient with sinus rhythm referred by Ramuschkat et al. [21].

ADMA directly inhibits NOS activity, whereas SDMA indirectly influences NO formation interacting with the transport of L-arginine, which may lead to an intracellular depletion of L-arginine, causing endothelial dysfunction [22].

Here a lower median L-arginine plasma concentration was observed $24 \mathrm{~h}$ after stroke than in our previous study measured in the serum at the same time point. In addition, more patients with mild severity (mean of NIHSS on admission: $6.3 \pm 4.8$ ) were recruited in this cohort compared to our previous study population (mean of NIHSS on admission: $11.5 \pm 5.4)$ [17].

Regarding the long-term outcome, here we found that higher plasma L-arginine level; L-arginine/ADMA and L-arginine/SDMA ratios at 24 post-stroke hours were associated with worsening mRS during the first 6 months after the onset of stroke. However, no significant correlation was observed between improved and worsened group, only a trend was found in the ADMA concentration between the two groups in this cohort (median: 0.58 , IQR: $0.46-0.8$ vs. $0.7,0.59-1.04$ ). Our data indicate that lower L-arginine level, lower L-arginine/ADMA and L-arginine/ SDMA ratio at $24 \mathrm{~h}$ is associated with higher mRS on discharge, i.e., worse outcome on the short-term; but these patients improve on the long-term resulting in association of lower L-arginine/SDMA with improving mRS. Similarly, patients with lower L-arginine/ADMA ratio improves on the long-term. In contrast, patients with higher L-arginine, L-arginine/ADMA and high L-arginine/SDMA and lower discharge mRS improve fast, but will not improve or may even worsen by 6 months indicating that its biological effect is time-dependent. Early elevation of $\mathrm{L}$-arginine in the acute phase of ischemic stroke may have a beneficial influence on cerebral perfusion by increasing NO, thus may be protective in the acute phase of ischemic stroke [17]. Among healthy individuals with a low risk of cardiovascular disease, those with plasma ADMA levels above $0.71 \mu \mathrm{mol} / \mathrm{L}$ had a higher risk of future cardiovascular and cerebrovascular events, as compared to those with plasma ADMA levels below [23]. Pikula et al. [24] observed that silent brain infarcts in MRI occur more frequently in subjects in the upper three age-specific quartiles of plasma ADMA concentrations compared to the lowest quartile. Plasma ADMA levels in the acute stage of stroke independently predicted adverse outcome at 90 days. Similarly, an increased plasma ADMA concentration was found here in patients who are not improved by 6 post-stroke months. ADMA levels $\geq 0.566 \mu \mathrm{mol} / \mathrm{L}$ at day 3 and $\geq$ $0.530 \mu \mathrm{mol} / \mathrm{L}$ at day 7 after symptom onset were independent predictors of an unfavorable outcome [25]. Infusion of ADMA increased vascular stiffness and decreased cerebral perfusion in healthy subjects [26]. We observed the elevation of ADMA and L-arginine at 24 post-stroke hours in patients with lower mRS on the short-term. We hypothesize that the detrimental effect of ADMA may be antagonized by an increased L-arginine level as a regulatory mechanism resulting in increased NO production and vasodilatation as we stated in our previous work [17]. Clinical studies found evidence that increased ADMA levels are associated with a higher risk of cerebrovascular events [27]. Based on our findings, an increased systemic concentration of ADMA less likely to be the result of acute stroke, rather a preceding factor contributing to cerebrovascular events. In accordance with this, coincidence in both, 
lower level of ADMA and L-arginine, suggests a lower vascular risk and a better outcome in the long term.

In our cohort, we found almost the same prevalence of smokers among patients with improving and worsening mRS. In contrast, more smokers were found in the unchanged outcome group showing a trend that may reflect ischemic preconditioning, but the difference was not significant. In previous studies, higher ADMA level was observed in smokers compared to non-smokers [28]. This connection is confirmed in our study; nevertheless it was not associated with worse outcome. Theoretically, smoking status combined with other cardiovascular risk factors increases circulating ADMA level and may affect negatively the restoration of ischemic damage. ADMA was positively associated with thrombo-inflammatory markers in the acute phase of ischemic stroke [29].

\section{Conclusion}

In conclusion, our cross-sectional data indicate that patients with high L-arginine plasma concentration $24 \mathrm{~h}$ after stroke improve fast, but will not improve further by 6 months suggesting that these patients might be in various stage of atherosclerosis. In addition, the beneficial effect of L-arginine seems time-dependent [8]. In contrast, lower $\mathrm{L}$-arginine/SDMA ratio is associated with worse outcome on the shortterm, but these patients may improve on the long-term.

There are some limitations of our study: (i) low number of enrolled patients decreased the power of this study and precluded detailed multivariate analysis; (ii) lack of follow up imaging study in all patients tackled us to explore relationship between penumbral or hypoperfused area and markers; and (iii) using single measurement of L-arginine pathway metabolites at 24 post-stroke hours, we were not able to explore association between change in concentrations of markers and functional outcome here.

\section{References}

1. Murray CJ, Vos T, Lozano R, Naghavi M, Flaxman AD, et al. (2012) Disabilityadjusted life-years (DALYs) for 291 diseases and injuries in 21 regions, 19902010: A systematic analysis for the global burden of disease study 2010. Lancet 380: 2197-2223.

2. Ovbiagele B, Goldstein LB, Higashida RT, Howard VJ, Johnston SC, et al. (2013) Forecasting the future of stroke in the United States: A policy statement from the American Heart Association and American Stroke Association. Stroke 44: $2361-2375$

3. Bustamante A, Simats A, Vilar-Bergua A, García-Berrocoso T, Montaner J, et al. (2016) Blood/brain biomarkers of inflammation after stroke and their association with outcome: From C-reactive protein to damage-associated molecular patterns. Neurotherapeutics 13: 671-684.

4. Habets KL, Huizinga TW, Toes RE (2013) Platelets and autoimmunity. Eur J Clin Invest 43: 746-757

5. Qu Z, Chaikof EL (2010) Interface between hemostasis and adaptive immunity. Curr Opin Immunol 22: 634-642.

6. Schulze F, Carter AM, Schwedhelm E, Ajjan R, Maas R, et al. (2010) Symmetric dimethylarginine predicts all-cause mortality following ischemic stroke. Atherosclerosis 208: 518-523.

7. Lüneburg N, von Holten RA, Töpper RF, Schwedhelm E, Maas R, et al. (2012) Symmetric dimethylarginine is a marker of detrimental outcome in the acute phase after ischaemic stroke: Role of renal function. Clin Sci (Lond) 122: 105111.

8. Worthmann H, Chen S, Martens-Lobenhoffer J, Li N, Deb M, et al. (2011) High plasma dimethylarginine levels are associated with adverse clinical outcome after stroke. J Atheroscler Thromb 18: 753-761.

9. Jauch EC, Saver JL, Adams HP Jr, Bruno A, Connors JJ, et al. (2013) Guidelines for the early management of patients with acute ischemic stroke: A guideline for healthcare professionals from the American Heart Association/ American Stroke Association. Stroke 44: 870-947.
10. European Stroke Organisation (ESO) Executive Committee, ESO Writing Committee (2008) Guidelines for management of ischemic stroke and transient ischemic attack 2008. Cerebrovasc Dis 25: 457-507.

11. Adams HP Jr, Bendixen BH, Kappelle LJ, Biller J, Love BB, et al. (1993) Classification of subtype of acute ischemic stroke. Definitions for use in a multicenter clinical trial. TOAST. Trial of org 10172 in acute stroke treatment. Stroke 24: 35-41.

12. Cohen J, Brun-Buisson C, Torres A, Jorgensen J (2004) Diagnosis of infection in sepsis: An evidence-based review. Crit Care Med 32: S466-S494.

13. Borgeraas H, Hertel JK, Svingen GF, Pedersen ER, Seifert R, et al. (2016) Association between body mass index, asymmetric dimethylarginine and risk of cardiovascular events and mortality in Norwegian patients with suspected stable angina pectoris. PLoS One 11: e0152029.

14. Barber PA, Demchuk AM, Zhang J, Buchan AM (2000) Validity and reliability of a quantitative computed tomography score in predicting outcome of hyper acute stroke before thrombolytic therapy. ASPECTS study group. Alberta stroke programme early CT score. Lancet 355 : 1670-1674.

15. The National Institute of Neurological Disorders and Stroke rt-PA Stroke Study Group (1995) Tissue plasminogen activator for acute ischemic stroke. N Engl J Med 333: 1581-1587.

16. Nonaka S, Tsunoda M, Imai K, Funatsu T (2005) High-performance liquid chromatographic assay of NG-monomethyl-Larginine, NG,NG-dimethylL-arginine, NG,NG'-dimethyl- L-arginine using 4-fluoro-7-nitro-2,1,3benzoxadiazole as a fluorescent reagent. J Chromatogr A 1066: 41-45.

17. Molnar T, Pusch G, Papp V, Feher G, Szapary L, et al. (2014) The L-arginine pathway in acute ischemic stroke and severe carotid stenosis: Temporal profiles and association with biomarkers and outcome. J Stroke Cerebrovasc Dis 23: 2206-2214.

18. Horecka A, Szpetnar M, Hordyjewska A, Babula D, Golab P, et al. (2016) Actylise treatment does not influence nitric oxide metabolites serum level. Pharmacol Rep 68: 598-600.

19. Worthmann H, Martens-Lobenhoffer J, Joumaah M, Li N, Lichtinghagen R, et al. (2013) Asymmetric dimethylarginine in response to recombinant tissue-type plasminogen activator and erythropoietin in acute stroke. Stroke 44: 21282133.

20. Wanby P, Teerlink T, Brudin L, Brattström L, Nilsson I, et al. (2006) Asymmetric dimethylarginine (ADMA) as a risk marker for stroke and TIA in a Swedish population. Atherosclerosis 185: 271-277.

21. Ramuschkat M, Appelbaum S, Atzler D, Zeller T, Bauer C, et al. (2016) ADMA subclinical changes and atrial fibrillation in the general population. Int $\mathrm{J}$ Cardio 203: 640-646.

22. Closs El, Basha FZ, Habermeier A, Förstermann U (1997) Interference of $\mathrm{L}$-arginine analogues with $\mathrm{L}$-arginine transport mediated by the $\mathrm{y}+$ carrier hCAT-2B. Nitric Oxide 1: 65-73.

23. Leong T, Zylberstein D, Graham I, Lissner L, Ward D, et al. (2008) Asymmetric dimethylarginine independently predicts fatal and nonfatal myocardial infarction and stroke in women: 24 year follow-up of the population study of women in Gothenburg. Arterioscler Thromb Vasc Biol 28: 961-967.

24. Pikula A, Böger RH, Beiser AS, Maas R, DeCarli C, et al. (2009) Association of plasma ADMA levels with MRI markers of vascular brain injury: Framingham offspring study. Stroke 40: 2959-2964.

25. Worthmann H, Chen S, Martens-Lobenhoffer J, Li N, Deb M, et al. (2011) High plasma dimethylarginine levels are associated with adverse clinical outcome after stroke. J Atheroscler Thromb 18: 753-761.

26. Kielstein JT, Donnerstag F, Gasper S, Menne J, Kielstein A, et al. (2006) ADMA increases arterial stiffness and decreases cerebral blood flow in humans. Stroke 37: 2024e9

27. Chen S, Li N, Deb-Chatterji M, Dong Q, Kielstein JT, et al. (2012) Asymmetric dimethyarginine as marker and mediator in ischemic stroke. Int J Mol Sci 13: 15983-16004.

28. Sobczak A, Goniewicz ML, Szoltysek-Boldys I (2009) ADMA and SDMA levels in healthy men exposed to tobacco smoke. Atherosclerosis 205: 357-359.

29. Molnar T, Pusch G, Nagy L, Keki S, Berki T, et al. (2016) Correlation of the $\mathrm{L}$-arginine pathway with thrombo-inflammation may contribute to the outcome of acute ischemic stroke. J Stroke Cerebrovasc Dis 25: 2055-2060. 